\title{
Generic Battery Model based on a Parametric Implementation
}

\author{
R.Prieto, J.A.Oliver, I. Reglero and J.A.Cobos \\ Universidad Politécnica de Madrid \\ Centro de Electrónica Industrial (CEI) \\ c/ José Gutiérrez Abascal, 2 \\ 28006 Madrid. SPAIN. \\ e-mail: roberto.prieto@upm.es
}

\begin{abstract}
Batteries are a common element used in many electronic applications. Therefore, the analysis and simulation of these applications requires a battery model in order to validate the behavior of the whole system. Since batteries are based on different technologies, a modeling approach valid for any technology is a potential good alternative. Since there are similarities among the different technologies, it is possible to address the modeling of batteries as generic energy storage elements with particular differences. This work presents a battery model valid for different technologies based on a parametric implementation.
\end{abstract}

\section{INTRODUCTION}

The main motivation of this work is based on the need of having an accurate and versatile battery model valid for system level electrical simulations. There is no need to talk about the many applications where batteries are used. A design process of all these applications based on simulations requires a battery model. Since batteries are based on different technologies, it is needed to develop a different model for each of those technologies. This work presents a modeling approach that is based on a general structure that is valid for different technologies.

The idea is to develop a general model with different blocks, identifying different functions in the batteries that are represented for each block. These functions are like the charge and discharge process, the temperature dependency, etc. Since these functionalities depend on the technology, the equations and parameters of each of those blocks should be determined for each technology and each battery.

The general modeling approach, the structure of the model and the way to extract the parameters will be presented in this work, including the validation process that has been carried out. One of the goals of the model is to use parameters that can be easily obtained from manufacturer data books or from simple tests. Another important feature of the model is that it is "scalable" in the sense that different "behaviors" can be included depending on the accuracy or information that the model should reproduce. Another advantage of this approach is that the model can be implemented even if part of the information is not available from the data books, such as the discharge curves or the internal resistance.

The model has been validated with experimental results and manufacturer data. Four different technologies have been tested (Li-ion, Lead-acid, Ni-MH, Ni-Cd), with transient and constant charge and discharge. 


\section{MODELING APPROACH}

Figure 1 shows the input parameters for the proposed model. The battery model is represented by the grey element at the center and the inputs are the different yellow boxes. As mentioned before, the model is based on several blocks that represent different parts of the battery behavior. Each block has different representations (equations) for each technology and the appropriate equations are internally selected depending on the technology that is selected through an input parameter (technology block). In particular, the model has been implemented for four technologies: LithiumIon, Ni-Mh, Ni-Cd and $\mathrm{Pb}$.

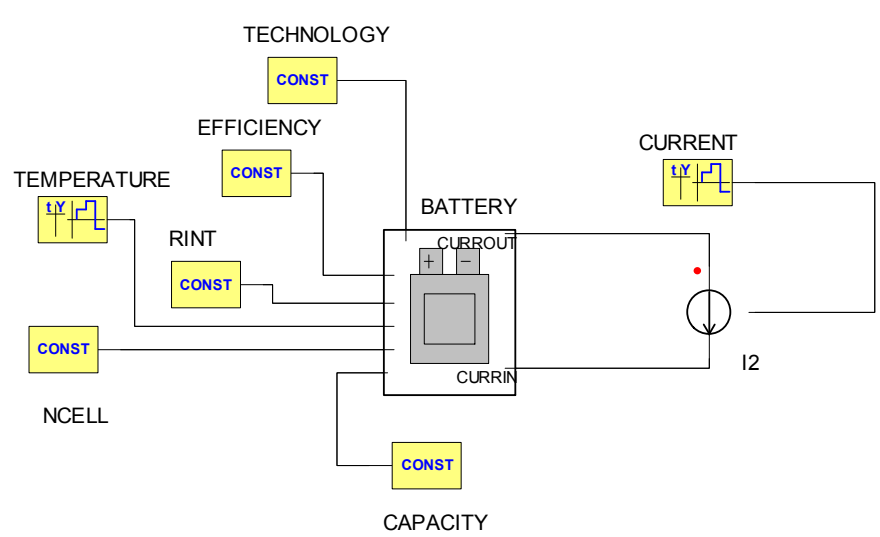

Figure 1. Inputs parameters (yellow boxes) of the battery model (grey element)

The rest of the blocks represent the efficiency, temperature, internal resistance, number of cells, capacity and conservative or non-conservative current.

Since the model is based on equations, it can be implemented in any simulator. An overview of the equations of the model will be presented in the next section, but it can be anticipated that the equations are not complex and this yields to fast computation times: simulations of $20 \mathrm{~h}$ take about 15 seconds.

The different technologies are represented by the following effects:

- Charge and discharge effects (State of charge function: $\mathrm{f}(\mathrm{SOCefect}))$

- Internal resistance as a function of the temperature and the current: $\mathrm{RT}(\mathrm{T}(\mathrm{t}), \mathrm{i}(\mathrm{t}))$

- Transfer function: $\mathrm{G}(\mathrm{Ti})$

These three features are different for each technology, and the equations used to characterize them are tailored for each technology.

Once the equations that represent the behavior are defined, the parameters of those equations are extracted by interpolation-fitting of real battery curves.

Figure 2 shows the block diagram of the proposed battery model that corresponds to the internal description of the grey element in figure 1

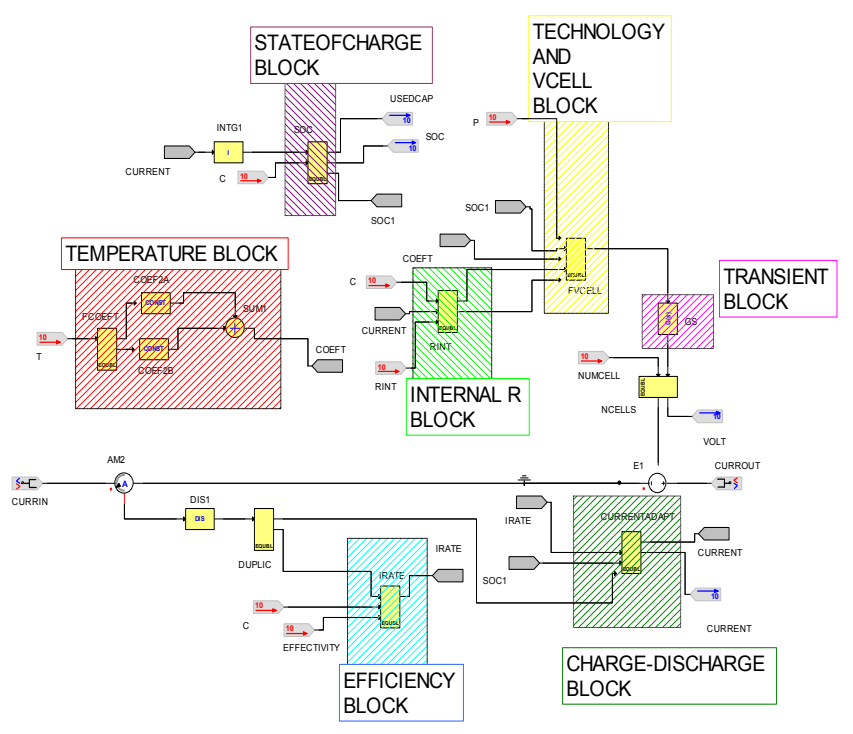

Figure 2. Structure of the proposed battery model 


\section{MODEL EQUATIONS}

This section presents the description of the model.

The inputs that have been selected in order to extract the proposed model are: instantaneous current $i(t)$, instantaneous temperature $\mathrm{T}(\mathrm{t})$, nominal capacity (Cnom), number of cells (n), internal resistance (Ri) and technology (p). The efficiency of the battery during the charge or discharge processes is represented by the loss factor $(\varepsilon)$ that depends on the temperature, and it is given by equations (1).

$$
i_{\text {rate }}(t)=\frac{i(t)}{C_{\text {nom }}}\left(1+\varepsilon_{C}(T)\right)
$$

As can be extracted from equations (1), a constant discharge of $1 \mathrm{C}$ with $\varepsilon=0.1$ would cause $10 \%$ of losses, that means that the actual capacity of the battery will be $10 \%$ reduced. The same procedure can be extended to the charge process, the battery would be charged to $90 \%$.

The way to obtain this parameter is to measure the final capacity used (Cf) in Ah obtained with 1C discharge rate, and to compare it with the nominal capacity given by the manufacturer (Cnom), as shown in equation (2)

$$
\varepsilon_{C}(T)=\frac{C_{\text {nom }}-C_{f}(T)}{C_{\text {nom }}}
$$

Once this parameter is defined the next step is to define the state of discharge SOD, as shown in equation (3)

$$
S O D(t)=\int_{t_{0}}^{t} I_{\text {rate }}(t) d t
$$

The resistance of the battery can be seen as a combination of an internal resistance (Ri) and a temperature depending resistance $(\mathrm{RT}(\mathrm{T}(\mathrm{t}))$ (equation (4)). Both parameters depend on the technology used, but they are different for each particular battery, as shown in figure 3

$$
R=R_{i}+R_{T}(T)
$$

Li-Ion Discharge Characteristics

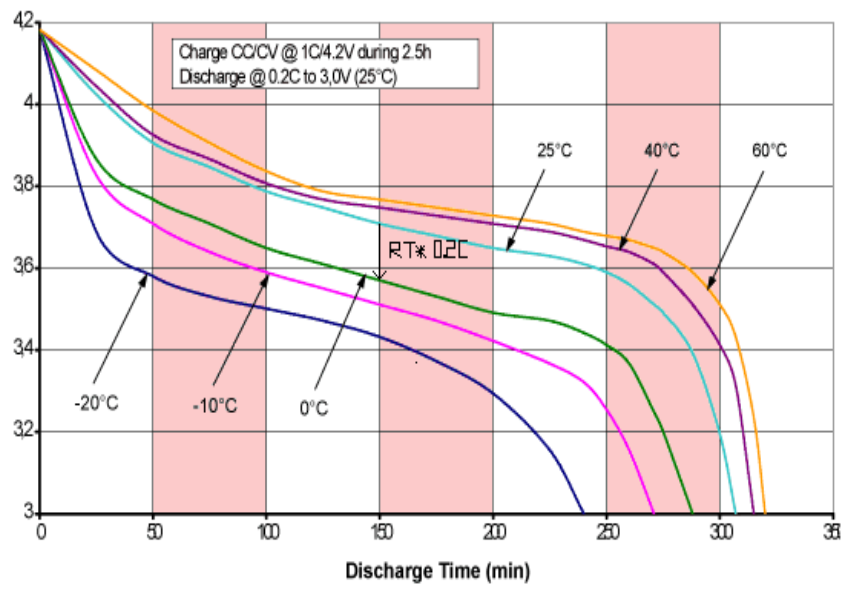

Typical discharge profiles at $+20^{\circ} \mathrm{C}$

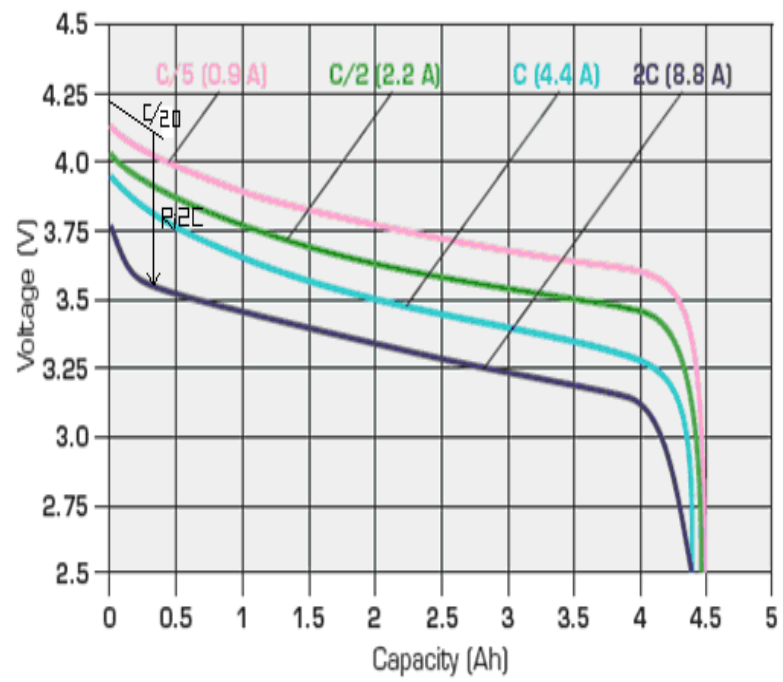

Figure 3. Discharge curves for different technologies and different batteries

The output voltage depends on the state of charge, the resistance and the current, as shown in equation (5).

$$
V_{0 \_ \text {ideal }}(t)=f\left(S O D_{e f}(t, T)\right)-R i(t)
$$


The transient response is calculated through the transfer functions shown in (6)

$$
V_{0}(s)=G(s) \cdot V_{0 \_ \text {ideal }}(s)
$$

The transfer function presented in (7) has been selected in order to represent the transient response.

$$
G(s)=\frac{1}{1+\tau_{i} s} \mathrm{G}(\mathrm{s})=1 /\left(1+\mathrm{Ti}^{*} \mathrm{~s}\right)
$$

Finally, the voltage obtained by the model is shown in (8) where $n$ is the number of serial cells

$$
V_{b a t}(t)=n_{\text {cells }} \cdot V_{0}(t)
$$

\section{MODEL VALIDATION}

The model has been validated comparing the model behavior with real tests. Static and transient tests have been performed in order to check the model, and those tests have been done with two different technologies (Li-Ion and $\mathrm{Pb}$ ). A constant discharge test of a Li-Ion battery $(1100 \mathrm{mAh} 3.7 \mathrm{v})$ is shown in figures 4 and 5 . Figure 4 shows the $0.08 \mathrm{C}$ current case and figure 5 shows the $0.28 \mathrm{C}$ current case. It can be seen that the model fits pretty well with the experimental measurements.

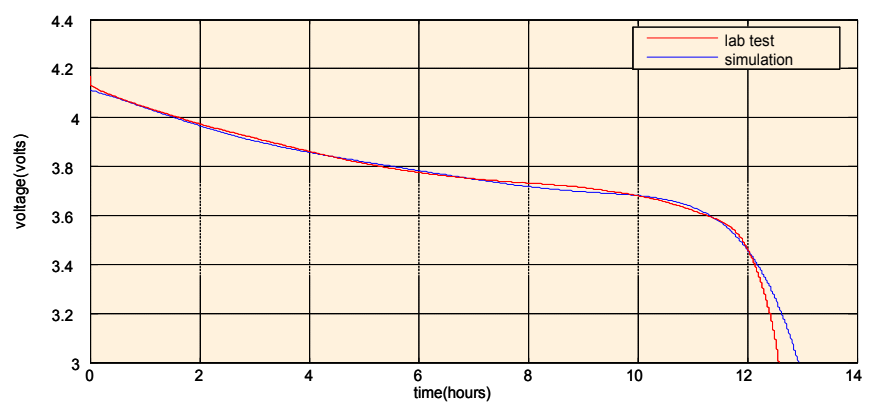

Figure 4. Discharge curve. Output voltage omparison between simulation (blue) and experimental results (red). Li-Ion battery 0.08 C current

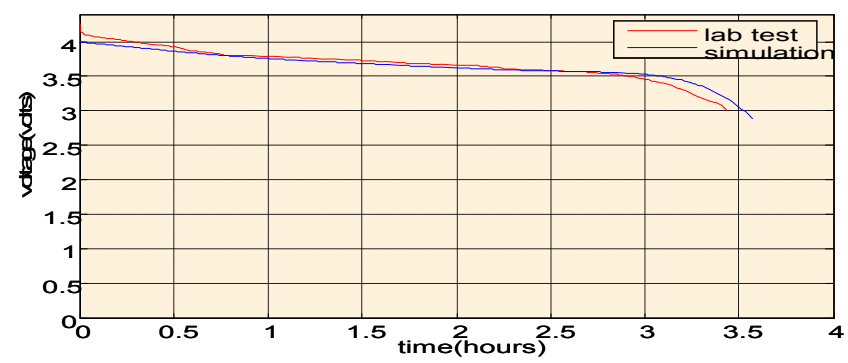

Figure 5. Discharge curve. Output voltage omparison between simulation (blue) and experimental results (red). Li-Ion battery 0.28 C current

\section{CONCLUSIONS}

This works presents a parametric model of batteries with the following features:

- It is based on a block structure where each block represents a different part of the behavior of the battery. This makes possible a parametric implementation based on different equations for each technology. The model selects the appropriate equations depending on the technology. Therefore, a simple generic model is valid for different battery technologies

- The model is implemented based on equations. This makes possible the use of the model in different simulation platforms

- The parameters of the model can be obtained from data-books or simple tests.

- The model is "scalable" in the sense of the addition of blocks that represents the behavior of different effects. Therefore, the model can be more or less complex but using the same structure. The information is not required or the input data is not available the model can be simplified removing part of the behavior in a very simple way. 


\section{REFERENCES}

[1] Data-books Saftbatteries, Bipowerusa, Powerstream, Probattery, Reapsystems)

[2] "Energetical modelling of lithium-ion battery discharge and relaxation". Urbain, M. Rael, S. Davat, B. Desprez, P. GREEN-INPL-CNRS, Vandceuvre-les-Nancy; Power Electronics Specialists Conference, 2008. PESC 2008. IEEE Date: 15-19 June 2008 On page(s): 3628-3634

[3] "An Enhanced Dynamic Battery Model of LeadAcid Batteries Using Manufacturers' Data" Nosh K. Medora Alexander Kusko. Telecommunications Energy Conference, 2006. INTELEC '06. 28th Annual International Date: Sept. 2006 On page(s): 1-8

[4] "Hybrid Advanced Power Sources (HAPS) Project". LIJUN GAO, ROGEL DOUGAL SHENGYI LIU. VTB Annual Review 2002

[5] "Accurate electrical battery model capable of predicting runtime and I-V performance". Min Chen Rincon-Mora, G.A. Sch. of Electr. \& Comput. Eng., Georgia Inst. of Technol., Atlanta, GA, USA; Energy Conversion, IEEE Transaction on. Date: June 2006 Volume: 21, Issue: 2 On page(s): 504- 511

[6] "A Generic Battery Model for the Dynamic Simulation of Hybrid Electric Vehicles". Tremblay, O. Dessaint, L.-A. Dekkiche, A.-I. Electr. Eng. Dept., Univ. du Quebec, Montreal, QC; Vehicle Power and Propulsion Conference, 2007. VPPC 2007. IEEE Date: 9-12 Sept. 2007 On page(s): 284289 